\title{
Correlational analyses of explicit and implicit memory performance
}

\author{
PIERRE PERRUCHET and PATRICE BAVEUX \\ Université de Paris V(René Descartes), Paris, France
}

\begin{abstract}
Sixty-four subjects were administered two tests of explicit memory (selective recall and recognition) and four tests of implicit memory (identification in a perceptual clarification procedure, word-fragment completion, tachistoscopic identification, and anagram solution). Each test drew on a different subset of a long list of previously displayed words. Although the four implicit memory tests showed sizable priming effects, correlational and factor analyses showed striking dissociations. On the one hand, performance on the perceptual clarification procedure and word-completion tests were related to one another, as well as to recall and recognition. On the other hand, performance on tachistoscopic identification and anagram solution were related to one another, but not to the measures for the other tasks. A framework is proposed to reconcile these new results with current knowledge on the explicit/implicit memory distinction, based in particular on studies of amnesic subjects. It is argued that a small number of tasks, especially tachistoscopic identification, may serve as relatively uncontaminated and ubiquitous indicators of implicit memory. However, explicit remembering could affect performance in so-called implicit memory tasks that allow for a strategy of controlled selection of candidate responses from accumulating cues, in experimental conditions that make the explicit remembering of relevant events possible.
\end{abstract}

Studies of human memory have traditionally focused on tests such as free recall, cued recall, and recognition, which require the explicit recollection of items through some sort of directed controlled search into stored information. However, it has become clear in recent years that effects of memory can also be observed through changes in performance on identification or production tasks involving previously studied stimuli, without the explicit retrieval of these stimuli being necessary. For example, when the initial event is the reading or the hearing of verbal items, subsequent modification in the processing of these items has been reported for reading performance (Moscovitch, Winocur, \& McLachlan, 1986), lexical decision (Carroll \& Kirsner, 1982), verbal association (Cofer, 1967), homophone spelling (Eich, 1984), anagram solution (Dominowski \& Ekstrand, 1967), tachistoscopic identification (Jacoby \& Dallas, 1981), identification in a perceptual clarification procedure (Feustel, Shiffrin, \& Salasoo, 1983), and word completion (Tulving, Schachter, \& Stark, 1982). Following Graf and Schachter (1985), the term implicit memory will be used here to designate the form of memory underlying such effects.

A striking argument in favor of the independence between conventional, or explicit, and implicit memory

This article is based in part on the second author's master's thesis, which was done at the Université René Descartes of Paris under the direction of the first author. The study was supported by the CNRS (UA 656), Université René Descartes, EPHE ( $3^{\circ}$ section), and CNAM (Service de recherches de l'INOP). The authors thank R. W. Proctor, N. Slamecka, and two anonymous reviewers for the useful suggestions they provided on an earlier version of this manuscript. Requests for reprints should be sent to Pierre Perruchet, Laboratoire de Psychologie Différentielle, 28 rue Serpente, 75006 Paris, France. comes from studies on amnesic patients. A number of experiments have shown that the effect, on amnesic patients and normal subjects, of previously displayed words is comparable in word completion tests (e.g., Graf, Squire, \& Mandler, 1984), reading tasks (Moscovitch et al., 1986), and homophone spelling (Jacoby \& Witherspoon, 1982), despite impaired recall or recognition of these words in amnesic patients (see review in Squire \& Cohen, 1984). Such results suggest that the neurological systems underlying implicit memory performance may remain intact even when the brain regions subtending explicit memory may be damaged. However, this kind of evidence, based on pathological disorders, needs to be corroborated by investigations on normal subjects.

The degree of independence between explicit and implicit memory in normal subjects has been assessed by comparing the behavioral laws regulating each type of performance and by evaluating stochastic relationships between these performances. Most authors emphasize that the results obtained by both methods converge to support clear-cut independence. We argue that this conclusion may be unwarranted.

There are a number of differences between regularities in explicit and implicit memory. In particular, a number of experiments have shown that manipulations intended to change level of processing during initial encoding have no influence on subsequent expression of implicit memory, whereas they have marked effects on explicit memory performance (Carroll, Byrne, \& Kirsner, 1985; Dark, Johnston, Myles-Worsley, \& Farah, 1985; Graf \& Mandler, 1984; Graf, Mandler, \& Haden, 1982; Jacoby \& Dallas, 1981; Ohta, 1984). Another acknowledged divergence is that decay from implicit 
memory appears to be a slower process than decay from explicit memory. For example, Feustel et al. (1983) reported that identification of a word in a perceptual clarification test was impaired only slightly when the number of items occurring between the previous exposure and the test went from 1-5 to $120-130$, in contrast to a sharp decrement in recognition performance. Tulving et al. (1982) observed no reduction in priming as assessed by a word-fragment completion test over a 7-day interval, although there was a sizable reduction in recognition (see also Dannenbring \& Briand, 1982; Jacoby, 1983; Jacoby \& Dallas, 1981; Komatsu, 1985; Nelson, 1978; Scarborough, Cortese, \& Scarborough, 1977).

However, these dissociations must be set in a more general context, which may be dominated by commonalities. In all his pioneering works, Ebbinghaus measured memory through the method of saving in relearning, which is typically an implicit memory task. Slamecka (1985) correctly pointed out that all of the independent variables Ebbinghaus investigated have by now been reexamined in explicit memory tasks, with roughly equivalent results. Moreover, even the divergencies cited in the previous paragraph have empirical and theoretical limitations.

At the empirical level, it is worth noting that a number of exceptions detract from the generality of the divergencies. Dark et al. (1985), Forster and Davis (1984), Logan (1985), and Oliphant (1983) showed in various ways that implicit memory can be affected by the nature of the initial encoding or by the orientation of attention during initial exposure to stimulation. For example, Oliphant (1983) showed that lexical decisions were not speeded up when the target words had been previously embedded either in a short preexperimental questionnaire or in instructions, instead of being displayed in the first part of the experimental session, as is standard procedure. With respect to the rate of decay over time, Salasoo, Shiffrin, and Feustel (1985, Experiment 3) reported that priming assessed by a perceptual clarification procedure disappeared after a lapse of one year, although recognition was still above chance (see also Jacoby, 1983; Sloman et al., 1988).

A more theoretical objection stems from the fact that none of the alleged empirical dissociations consist of actual oppositions. As a general rule, implicit tests exhibit some degree of persistence in memory, whereas explicit tests do not. This kind of difference may be parsimoniously imputed to the differential sensitivity of both measures to small amounts of stored information. In short, framing the question of dissociation in terms of behavioral laws cannot provide decisive arguments in favor of a genuine independence of the explicit and implicit memory forms. There is no convincing evidence that these dissociations are greater than those between measures of recall and recognition (Jacoby, 1983).

Let us turn now to the analysis of contingencies between explicit and implicit memory measures. A demonstration of stochastic independence between primed and explicitly retrieved items would be crucial in ruling out an index- sensitivity account for the differences in regularities. A number of investigators have submitted subjects successively to an implicit and an explicit memory task involving the same previously displayed items. When the implicit memory test precedes recognition, a positive correlation is regularly obtained between performances: primed items tend to be recognized more often than unprimed items (Jacoby \& Witherspoon, 1982; Light, Singh, \& Capps, 1986; Tulving et al., 1982; see also Clifton, 1966 , with a recall measure). This result suggests that there is some degree of relationship between the underlying processes. However, Tulving et al. (1982) disregarded this interpretation and explained the correlation by the fact that the priming task constitutes an additional opportunity to study the primed items, thus facilitating their subsequent recognition. When recognition precedes the implicit memory tasks, a similar correlation has been found with nonword items (Jacoby \& Witherspoon, 1982, Experiment 1), but performances with word items always show stochastic independence (Jacoby \& Witherspoon, 1982; Light et al., 1986; Tulving et al., 1982).

Although the latter result is commonly interpreted as lending strong support to independent memory forms, its methodological adequacy can be questioned. Presentations of words on recognition tests must exert strong beneficial effects upon their immediately subsequent identification or production in the implicit memory task, whether or not these words are recognized as old. Shimamura (1985) used a hypothetical example to show how this effect dramatically reduced a simulated initial dependency between recognized and primed items and made the initially significant chi-square test nonsignificant. Moreover, Shimamura's demonstration may have underestimated the problem created by the successive testing of the same items because of the assumption that the effect of the initial presentation was still apparent, supposedly through an unspecified process of summation with the effect of the more recent presentation. This assumption is somewhat unlikely, given that repetitions have a slight and unstable effect on implicit memory data (Jacoby \& Dallas, 1981, Experiments 4a and 4b; Graf \& Mandler, 1984, Experiment 2). In other words, the effect generated by the words embedded in the recognition test may entirely obliterate any residual effect of the initial presentation.

To dismiss the possibility of an artifact due to successive testing of the same items, Tulving (1985) claimed that positive dependency is regularly found between recall and recognition in similar designs. However, this argument is irrelevant, because recall and recognition tests are highly sensitive to repetition effects, and hence the influence of an earlier presentation would remain manifest despite the leveling action of a more recent one. Thus, both comparisons of behavioral laws and contingency analysis leave the issue of the independence between explicit and implicit memory data open to question, insofar as normal subjects are concerned.

Up to now, only relationships between explicit and implicit memory have been examined. This framing, as well as similar discussions in the current literature, gives the 
impression that the unity of each form of memory requires no further confirmation. In fact, although strong arguments support the fundamental unity of explicit (episodic) memory (e.g., Tulving, 1976; Underwood, Boruch, \& Malmi, 1978), evidence concerning implicit memory is far less substantial. On the one hand, a survey of the general literature tends to favor unity: there are no acknowledged systematic differences between the results from different implicit memory tasks. However, such an assessment is at best suggestive, because, as a rule, each investigator uses a single task, selected according to tacit criteria. Thus, the congruence of results across tasks may be evaluated only through interstudy, and often interlaboratory, comparisons-that is, with substantial confounding factors.

On the other hand, Moscovitch et al. (1986) reported an unpublished experiment, conducted by Witherspoon and Moscovitch, that showed stochastic independence between performances on word completion and tachistoscopic identification tasks. Their design involved the successive testing of the same items. However, any artifact of consecutive presentation should have favored stochastic dependence, for the very same reason that Tulving et al. (1982) put forward to account for the stochastic dependence between explicit and implicit memory tasks when the latter precede the former. Thus, the unpublished findings of Witherspoon and Moscovitch suggest that implicit memory may thus far have been wrongly postulated as homogeneous.

The present study was aimed at shedding additional light on the issues of both the unity and the independence of implicit memory. We used a correlational approach, but with subjects, rather than items, taken as the unit of analysis. This method avoids the previously mentioned methodological pitfall of successive testing of the same items, at the cost of using far more subjects and items than had been employed in previous studies.

The subjects were given six memory tasks, each bearing on a different subset of a long list of previously displayed words. There were two explicit memory tasks (selective recall and recognition), and four implicit memory tasks (identification in a perceptual clarification procedure, word-fragment completion, tachistoscopic identification, and anagram solution). The selective-recall task involved presentation of a category name; the subjects were to recall all members of that category that had occurred in the initial list. Selective recall, rather than free recall, was used to minimize interference with the other tasks; that is, a more conventional free-recall procedure inevitably would have led subjects to remember words involved in the other tasks.

The tasks capable of measuring implicit memory are numerous and somewhat heterogeneous. Because of the relatively small current body of theoretical knowledge regarding this form of memory, we made no attempt to select the implicit memory tasks according to controlled criteria. Instead, it appeared more informative to use well- known procedures. Mirror drawing (Kolers, 1976), face identification (Ellis, Young, Flude, \& Hay, 1987), and picture naming (Carroll et al., 1985) were not included because they involve material other than words, and lexical decision (Scarborough et al., 1977) was discarded because it involves a number of additional processing steps that are linked to decision processes (e.g., Lorch, 1986). The four tasks we finally adopted are the most commonly used of the remaining available implicit memory tasks.

Inferences about relationships between the processes underlying performance of the respective tasks were drawn from between-subjects product-moment correlations and subsequent factor analysis of correlations, according to a research strategy that has proven its heuristic value within the field of explicit memory (e.g., Geiselman, Woodward, \& Beatty, 1982; Underwood et al., 1978).

\section{METHOD}

\section{Subjects}

Sixty-four third-year university students majoring in psychology served as subjects, in partial fulfillment of a course requirement. They reported normal or corrected-to-normal vision, and were native French speakers.

\section{Materials}

The materials consisted of a pool of 220 words. All words were common nouns, five letters in length, without diacritical marks; they occur with low or medium frequency in French. The words were randomly assigned to experimental conditions, except for the recall and anagram tasks, as described below.

The words were displayed via the standard video unit of an Apple Ile microcomputer for their initial presentation and for the perceptual clarification procedure, the tachistoscopic identification task, and the recognition task. The characters were uppercase roman letters, thicker than the standard characters of the microcomputer. The vertical and horizontal visual angles subtended were approximately $.36^{\circ}$ and $3^{\circ}$ for an entire word.

For the word-fragment completion and anagram tasks, the words were typewritten on a blank sheet of paper in capital letters. The dimensions of a word were about $3.5 \times 26 \mathrm{~mm}$.

\section{Procedure}

In the initial (study) phase, the subjects were shown a list of 120 words. Each item was displayed for $3 \mathrm{sec}$, then the screen was cleared for $1 \mathrm{sec}$ before the appearance of the next item. The subjects were instructed to read the words silently; they were informed that this task would be followed by memory tests, but were discouraged from adopting mnemonic strategies such as chunking. The 120 words consisted of six randomly intermixed sets of 20 words, each set being assigned to a memory task. One set, consisting of 20 animal names that were used for the selective-recall task, was the same for all subjects. For the other sets, half of the subjects (Group A) studied the words that were presented as new words to the remaining subjects (Group B) in the subsequent memory tests, and vice versa. The subjects were alternately assigned to Groups $A$ and $B$ in their order of presentation.

As soon as the entire list had been read, the subjects were given a distractor task for $5 \mathrm{~min}$, which consisted of completing an individual test of field dependence adapted from the Group Embedded Figures Test (Witkin, Oltman, Raskin, \& Karp, 1971). Performances on this test were scored and included as a variable in 
the correlational analysis for exploratory purposes. However, the correlations with other variables were negligible, and will therefore receive no further mention.

All subjects then performed the six memory tasks in succession, in the same order: selective recall, perceptual clarification, word completion, tachistoscopic identification, anagram, and recognition. The explicit memory tasks (selective recall and recognition) are described first, to be consistent with the Results section.

\section{Explicit Memory Tasks}

Selective recall. The subjects were asked to recall the animal names that had previously been presented. The subjects wrote their responses on a blank sheet. Three minutes were allotted for this task. The number of correct responses was used as the dependent variable.

Yes-no recognition. A list of 40 words, composed of 20 old words and 20 new words, was displayed on the video screen. During this task, each subject held a small box, with each thumb resting on a key. The subjects were instructed to press the key corresponding to their preferred hand when a word was recognized as part of the study list, and the other key when a word was not. Although no constraints on time were imposed, the subjects were encouraged to respond within 2 or $3 \mathrm{sec}$. Their responses triggered screen clearing, and the next word appeared $2 \mathrm{sec}$ later. The main dependent variable was the number of hits minus the number of false alarms. Response latencies were also recorded, to ensure that between-subjects differences in speed-accuracy tradeoff would not undermine the validity of the main dependent variable.

\section{Implicit Memory Tasks}

The four implicit memory tasks had several features in common. In each, the subjects were shown lists of 40 words, composed of 20 old words and 20 new words pseudorandomly intermixed. The subjects were informed prior to each test that it would include words presented during the study phase of the experiment. However, in structions stressed responding with the first word that came to mind, without considering whether or not it had previously been presented. Difference scores between performances for old and new words were computed for all tasks.

Although details of the method did not exactly match those of any previously published study, the general procedures did not depart from common practice. There was, however, one exception. As a rule, investigators submit subjects to strictly identical conditions. For example, subjects have to complete a set of 40 word fragments within a set period of time. Suppose that Subject 1 completes 20 words and Subject 2 completes 5 words. Since the individual amount of priming is assessed by subtracting the new words from the old words out of those correctly completed, it follows that Subject 1 has a maximum difference score of 20 points (if the 20 completed words are old), and Subject 2 has a maximum difference score of 5 points (if the 5 completed words are old; the same result is obtained when a subject completes 35 words, if the 5 noncompleted words are new). More formally, the maximum score a subject can obtain may be assessed as $N / 2-|T-N / 2|$, where $N$ corresponds to the overall number of word fragments displayed and $T$ to the number of actually completed words; when $T$ departs from $N / 2$, the maximum difference score drops. This situation is obviously detrimental when using correlational analysis, for at least two reasons. First, the lowering of the maximum scores results in truncation of the range of the variables being correlated, thus artificially deflating the resulting correlations. Second, any individual difference score may be a function of the strength of the priming effect (as intended) or of the overall number of completed words (which is irrelevant). To maximize and homogenize the range of the difference scores, an effort was made to adjust conditions for each sub- ject in such a way that all subjects completed, identified, or solved approximately half of the lists.

The special characteristics of the specific implicit memory tasks were as follows.

Perceptual clarification procedure. This task was similar in nature to the tests used by Feustel et al. (1983), and, more precisely, to the "continuous threshold latency identification" version (see also Johnston, Dark, \& Jacoby, 1985). Each trial began with an instruction to the subject to initiate the trial by pressing a key. Then a word appeared embedded within a mask, which gradually vanished. The technique of masking differed from the method used in the abovementioned studies, but in a way that presumably did not change the nature of the tasks for the subjects. The masks were formed by superimposing dots in the virtual matrix enclosing the words, so that a point falling inside the boundaries of a letter (normally on) was off, and a point falling outside a letter (normally off) was on. The dot coordinates were randomized and varied for each word. The visual signal-to-noise ratio was progressively increased via a point-by-point on/off inversion of the mask, in a random order. A point was inverted each $20 \mathrm{msec}$. As soon as the word became clear enough to be identified, the subject pressed a key, which triggered the return of the complete mask, and simultaneously said the word aloud. Both the latency of the keypressing responses and the correctness of verbalizations were recorded. Responses with a latency above $3,500 \mathrm{msec}$ were eliminated. Final difference scores were computed from latency data conditionalized on correct responses.

Word-fragment completion. The list of $\mathbf{4 0}$ word fragments was presented on a sheet of paper. Each item consisted of three letters and two underlined blank spaces, and allowed only one legitimate completion. The subjects were told to write the two missing letters in each fragment, so that the resulting letter string formed a common, singular, meaningful noun. They were prompted to scan the word fragments freely and to do the easier items first. Testing was stopped when the subject had completed about half of the list.

Tachistoscopic identification. The general procedure was patterned after that of Jacoby and Dallas's (1981) experiments. The subject initiated each trial by pressing one of the two keys that also served for the recognition test. The keypressing triggered the appearance of two horizontal lines surrounding the location in which the word would be presented. After its presentation, the word was immediately replaced by a string of five percent symbols, which remained on the screen for $2 \mathrm{sec}$. The subjects were instructed to say aloud the word that had been presented. They were encouraged to give a response.

The main departure from Jacoby and Dallas's (1981) procedure concerned the duration of exposure of the flashed word. Presentation duration was adjusted for each subject so that about half of the items would be correctly identified. Adjustment was made during a preliminary session. A set of words, which were not presented elsewhere, were displayed for practice. The first word was presented for $140 \mathrm{msec}$; the presentation duration was decreased by $20 \mathrm{msec}$ every time a word was correctly identified. The duration selected for the main test list was the lowest obtained correct identification value. Thus, the duration was $20 \mathrm{msec}$ for some subjects, $40 \mathrm{msec}$ for others, and 60 msec for others (a 20-msec step corresponds to a TV frame in European norms).

Anagram solution. The general procedure for anagrams was similar to that used for word completion: anagrams were typed on a sheet of paper, and the subjects were instructed to scan the items freely. Words for the anagrams were selected from the initial pool of words so that only one solution could be generated for a given string of letters. Anagrams were formed by rearranging two letters in each word, without systematic rearrangement rules. The subjects were tested individually. The subjects were stopped when they had solved about half of the list. Each session lasted about $1 \mathrm{~h}$. 


\section{RESULTS}

\section{Mean Performance on Explicit Memory Tests}

The subjects recalled a mean of 5.53 animal names of the 20 initially presented. Although this mean rate is too low to be indicative of subjects' maximum discrimination, the scores were nevertheless spread over a broad scale and went from 1 to 14 correct responses. The recall scores of Groups A and B did not differ significantly (5.62 vs. $5.53, t=.25$ ).

In the recognition test, 1 subject, who pressed the yes button on each trial, was eliminated. Of the 63 remaining subjects, the mean rate of hits was 13.79 , and the mean rate of false alarms was 4.39 . Therefore, the mean final difference scores was 9.40 (with a range of 2 to 17). Unexpectedly, the performances of Groups $A$ and $B$ differed $[10.65$ vs. $8.09, t(61)=2.65, p<.02]$. This difference was not a matter of a speed-accuracy tradeoff, since latency of recognition was shorter for Group A than for Group B [1,074 vs. $1,342 \mathrm{msec}, t(61)=2.61, p<.02]$. This pattern of results suggests that differences must be imputed to the differential difficulty of the word lists.

\section{Mean Performance on Implicit Memory Tests}

One subject regularly failed to respond before full clarification in the perceptual clarification procedure, and another subject was unable to complete any word fragments. The performance of both subjects was eliminated from the data. The main results for the remaining subjects in the implicit memory tasks are shown in Table 1. A sizable priming effect was obtained on each of the four tasks. The performances of Groups A and B were highly similar: for each task, a significant priming effect was obtained when groups were analyzed separately, and the amount of priming did not differ between groups $(t<1$ in the four cases). This pattern of results shows that the overall priming effect obtained in each task was a genuine one.

Possible bias may have existed at the individual sub ject level for completion, tachistoscopic identification, and anagrams, because the maximum difference scores depended on the number of items correctly completed, identified, or solved (see Method section). Although special care was taken to avoid large between-subjects differences in the overall number of responses, there was some residual variation. To test for the impact of this variation on final difference scores, the latter were correlated with the individual maximum difference scores, computed as indicated in the Method section. Between-subjects correlations were .163 for completion, .018 for tachistoscopic identification, and -.005 for anagrams. These negligible values indicate that individual difference scores are valid estimates of implicit memory efficiency.

\section{Correlations, Multiple Correlations, and Factor Analysis}

All of the following analyses were performed separately for Group A, Group B, and total subjects. Because the individual group analyses yielded conclusions identical with those of the global analysis, only the latter is reported here.

The correlation matrix for the six memory tasks is shown in Table 2 . The sign of the correlations between the perceptual clarification procedure (which involves latency measures) and the other tasks (which involve accuracy measures) was inverted; thus, any positive correlation represents a true positive relationship, and any negative correlation represents a true negative relationship.

The strongest correlation (.501) was observed for recall and recognition. Of the implicit memory tasks, perceptual clarification and word completion, on the one hand, and tachistoscopic identification and anagrams, on the other hand, were significantly related $(p<.05)$. Unexpectedly, there was a trend toward a negative relationship between perceptual clarification and tachistoscopic identification $(.05<p<.10)$.

Correlations between explicit and implicit memory tasks were somewhat heterogeneous. Tachistoscopic identification was unrelated to explicit memory, whereas other implicit memory tasks were significantly correlated with recall and/or recognition. When recall and recognition are taken together, the multiple correlation with implicit memory tests decreases slightly from word completion $(R=.381, p<.01)$ to perceptual clarification $(R=.331$, $p<.05)$ to anagram solution $(R=.29, .05<p<.10)$. Conversely, when the implicit memory data are combined

Table 1

Mean Performance on the Four Implicit Memory Tests for New and Old (i.e., Displayed in the Study Phase) Words

\begin{tabular}{|c|c|c|c|}
\hline Measure & New Words & Old Words & $\begin{array}{c}\text { Differences } \\
\text { (Priming Effect) }\end{array}$ \\
\hline $\begin{array}{l}\text { Latency of identification in the perceptual } \\
\text { clarification procedure ( } \mathrm{msec} \text { ) }\end{array}$ & 2886 & 2766 & $t(62)=6, p<.001$ \\
\hline $\begin{array}{l}\text { Number of completed words } \\
\text { in word-fragment completion }\end{array}$ & 6.40 & 9.43 & $\begin{array}{c}3.03 \\
t(62)=10.4, p<.001\end{array}$ \\
\hline $\begin{array}{l}\text { Number of identified words } \\
\text { in tachistoscopic identification }\end{array}$ & 9.70 & 12.51 & $\begin{array}{c}2.81 \\
t(63)=7.28, p<.001\end{array}$ \\
\hline Number of solved anagrams & 7.01 & 8.73 & $\begin{array}{c}1.72 \\
t(63)=4.49, p<.001\end{array}$ \\
\hline
\end{tabular}


Table 2

Matrix of Intercorrelations Between the Tests of Explicit (Variables 1 and 2) and Implicit (Variables 3 to 6) Memory

\begin{tabular}{|c|c|c|c|c|c|c|c|c|}
\hline \multirow{2}{*}{\multicolumn{2}{|c|}{ Tests }} & \multicolumn{5}{|c|}{ Intercorrelations } & \multicolumn{2}{|c|}{ Factors } \\
\hline & & 1 & 2 & 3 & 4 & 5 & 1 & 2 \\
\hline 1 & Recall & & & & & & .596 & .088 \\
\hline 2 & Recognition & $.501 \dagger$ & & & & & .702 & .020 \\
\hline 3 & Clarification & $.276^{*}$ & $.297 *$ & & & & .529 & -.184 \\
\hline 4 & Completion & .161 & $.379^{*}$ & $.305^{*}$ & & & .471 & .279 \\
\hline 5 & Identification & -.065 & -.026 & -.249 & .135 & & -.139 & .606 \\
\hline 6 & Anagrams & $.253^{*}$ & -.005 & .017 & .246 & $.268^{*}$ & .166 & .515 \\
\hline
\end{tabular}

Note-Due to missing data, correlations were computed on 62,63 , or 64 subjects. The last two columns give the results of a factor analysis (principal axes with varimax rotation) performed on the matrix. $\quad{ }^{*} p<.05 . \quad t p<.001$.

as predictors in a multiple regression equation, they appear to be more closely related to recognition $(R=.431$, $p<.025)$ than to recall $(R=.380, .05<p<.10)$.

The correlations between the six variables were factor analyzed. We present results from a common factor model; a principal-factors analysis was also performed and led to the same basic conclusions. Communalities were initially estimated by square multiple correlations. Two factors were extracted. The absolute values of the off-diagonal residual covariances averaged to .064 ( $S D=$ .04). The right-hand columns in Table 2 show the saturations on the two orthogonal principal axes after five iterations and varimax rotation. Factor 1 , which accounted for $23.3 \%$ of the variance, involved recognition, recall, perceptual clarification, and word completion. Factor 2, which accounted for $12.5 \%$ of the variance, was clearly tied to tachistoscopic identification and anagram solution.

\section{DISCUSSION}

Some preliminary comments on the overall low values of the correlations displayed in Table 2 are in order. The actual relationships between variables may be far stronger than the coefficient values suggest. First, some of the distributions, especially the distribution of recall scores, were slightly skewed. Second, correlations with recognition may have been lowered by the probable difference in recognition task difficulty between the two groups of subjects. Beyond these specific and presumably moderate influences, there is a more powerful and general factor that may have lowered estimates of relationships: five of the six variables involved difference scores. Such scores are typically unreliable, because their error variance cumulates the error variances of each initial measure. This problem in the use of derived measures is inherent in the operational definition of implicit memory, which always involves a comparison of performances with and without a priming event. Thus, the discussion focuses upon the overall structure of relationships, rather than upon the strength of individual associations.

The major finding of the present study is that performance on tachistoscopic identification, and, to a lesser extent, on anagram solution, is distinct from performance on both the other implicit memory tasks and the explicit memory tasks, whereas the effects exerted by prior exposure in a perceptual clarification procedure and a wordcompletion task are associated with explicit memory performance. In the present study, tasks were administered in a fixed order for all subjects, as necessitated by the analysis of individual differences. This methodological constraint made it possible for the resulting correlational pattern to reflect position in the test sequence, rather than the characteristics of the specific tasks. However, it should be emphasized that all of the possible factors mediating mean performance (such as subjects' familiarity with the experimental setting, fatigue, strategies, or motivational changes) have no necessary concommitant effects on correlational data, which depends on the distribution of individual performances around the mean. The latter factors may indeed affect correlations if they exert different effects on different subjects in a consistent way. If such is the case, the correlation between two tasks would decrease with increasing distance between tasks, and hence should be largest when one task immediately follows the other. In line with this assumption, inspection of the data reveals that Tasks 1 and 2,2 and 3, and 4 and 5 (as numbered in Table 2) were significantly related. But other adjacent tasks ( 3 and 4, 5 and 6 ) were not correlated; moreover, reliable relations were found between far removed tasks ( 1 and 5,2 and 6,3 and 6), with the largest correlation in the matrix concerning the most distant tasks (1 and 6). Overall, this analysis provides little, if any, support for the claim that the correlational pattern was an artifactual consequence of the running order of the tasks, and calls for other kinds of interpretation.

\section{The Dissociation Between Implicit Memory Measures}

A sizable priming effect was present in all the implicit memory tasks. However, the correlational pattern suggests that previous exposure to the stimuli could have exerted an effect on performance through two quite different processes. Some kind of dissociation could be anticipated from the results of Witherspoon and Moscovitch (cited in Moscovitch et al., 1986); using a different method, these authors found stochastic independence between performances on word completion and tachistoscopic identification tasks. At first glance, 
however, the line of division revealed by the present largescale study is somewhat surprising. There were a priori formal similarities between word completion and anagrams on the one hand, and between perceptual clarification and tachistoscopic identification on the other hand; the latter were video-displayed, unfamiliar tasks, both of which required perceptual identification in degraded conditions, whereas the former tasks were pencil-and-paper, relatively familiar tasks, which involved production of words. That the empirical dissociation is orthogonal to the action of these somewhat trivial factors reinforces its potential value and interest.

The explanation we would like to introduce at this point to account for these findings stems from casual observations made during the running of individual experiments, as well as from introspective reports collected during informal postexperimental interviews. Consider first the two priming tasks that exhibited a trend toward a negative relation, namely, the perceptual clarification procedure and tachistoscopic identification. Despite their formal similarities, these tasks were generally processed in extremely different ways. In the perceptual clarification procedure, the subjects drew out relevant information bit by bit; some letters become identifiable slightly before others, and subjects could build hypotheses upon these cues to reach a final solution. Responses typically appeared to be the end products of hypothesis-testing, directed processing. In contrast, in tachistoscopic identification, the subjects typically identified the flashed words in an all-or-nothing, immediate fashion. They often reported that a given word was "not really read," but rather that it forced itself upon them, creating a new subjective experience. There was some kind of direct access to responses, rather than a progressive and more or less controlled selection of candidate words from accumulating cues.

Objective empirical evidence supporting this contention is, accordingly, very sparse. Nevertheless, a somewhat indirect confirmation is provided from an analysis of errors. Erroneous responses in perceptual clarification often consisted of words whose graphemic features were almost those of the words presented. By contrast, the subjects often said a word that had little or no relationship to the actual flashed word in tachistoscopic presentation. In order to quantify differences, the number of well-ordered letters that the pronounced and actually flashed words had in common were tabulated (e.g., the pronounced word VOLTAGE has three well-ordered letters in common with the flashed word VALVE: V, L or A, and E). Of the 692 recorded erroneous responses, the proportion of words with three or fewer letters correct was .289 in perceptual clarification and 644 in tachistoscopic identification. (This analysis, which sometimes required an arbitrary choice of the actual spelling of words, was conducted by a scorer uninformed of the experiment's design and objectives.) This striking difference points to the fact that subjects tend to access correct responses through a step-by-step construction in the first case, and via a more direct pathway in the second case.
With regard to the postulated processing strategies, there are reasons to associate perceptual clarification and word completion. The word fragments furnish a cue that may be successfully completed by a systematic, serial, controlled procedure, all the more so because it is often easy to guess whether the missing letters are consonants or vowels. Anagrams, on the other hand, can hardly be solved by a systematic scanning of possible solutions (recall that there are 120 arrangements of 5 letters). More probably, the solution "pops out" from a diffuse, undirected exploration, a feature that makes this task similar to tachistoscopic identification. The nearly significant correlation between word completion and anagrams suggests, nevertheless, that the strategies involved in these two tasks differ less than do the strategies involved in perceptual clarification and tachistoscopic identification.

Note that the foregoing discussion focuses on the general processing of the tasks, whereas the results here concern the effect of priming that these tasks make manifest. Both points are obviously related: The way a prior event exerts an action on a later task is tightly linked to processing modalities of this task. However, up to now no difference has been suggested regarding the priming effect per se, and we will return to this point later.

\section{The Relationships Between Explicit and Implicit Memory Measures}

The description of the relevant literature in the introductory section suggested that the independence between explicit and implicit memory is less clear-cut than has been previously claimed. In particular, extending a line of reasoning first developed by Shimamura (1985), we argued that prior evidence for stochastic independence could be an artifactual consequence of the successive testing of the same items. The results of the present experiment show that when a more suitable methodology is employed, explicit memory correlates to some extent with implicit memory.

Correlations between explicit and implicit memory performance can be accounted for in quite different ways. One hypothesis is that the two types of memory depend on the same general aptitude. All of the tests in the present experiment used verbal material. Given that most verbal tasks have positive loadings on an identical group factor in traditional psychometric studies, it may be argued that the observed correlations proceed from this common component. Although a conclusive rebuttal of this account would require further systematic investigation, a general criticism can be put forward. Implicit memory scores assess the effect of a prior event on a verbal task, and not verbal performance per se. Thus, they could be unconfounded with a putative general ability to perform verbaltype tasks. For instance, although the subject's ability to complete words may be verbal in nature, there is no empirical or logical basis for believing that the effect of a priming event on word completion also depends on verbal aptitude. 
A second hypothesis is that implicit memory mediates to some extent explicit memory performances. Jacoby (e.g., Jacoby \& Dallas, 1981) argued that subjects can detect the relative fluency of perception of previously studied items, and can use this feeling as a cue for judging these items as old in a recognition task. In support of this view, Johnston et al. (1985) showed that items that were identified quickly in a perceptual clarification procedure tended to be judged old in an immediately subsequent recognition judgment, regardless of their actual old or new status. This conceptualization leads to a prediction of positive correlations of implicit memory with recognition, but not with recall, the latter presumably depending on a directed search factor.

The present results show that global relationships of implicit memory are slightly stronger for recognition than for recall. However, recall was significantly related to performance on perceptual clarification and anagrams, and recognition scores were found to be independent of performance on anagrams and tachistoscopic identification. Jacoby's (e.g., Jacoby \& Dallas, 1981) argument fails to account for this pattern of results. This does not mean that it is wholly incorrect, but its relevance may be restricted to strictly specified conditions. Ease of perception could be detected and used as a valid cue only under a few favorable conditions, such as those described by Johnston et al. (1985) or Moscovitch et al. (1986). In more natural settings, it may be the case that relative perceptual fluency is unavailable to awareness, and that recognition relies upon other factors.

The correlation pattern obtained in the present study may be best explained by a third hypothesis, having to do with the influence of explicit memory upon implicit memory. It could be argued that this influence was reinforced by certain features of the instructions, thus limiting the generality of the results. Indeed, before the subjects were instructed to respond with the first word that came to mind, they were told that the test included words presented during the study phase of the experiment. This information, although included in the instructions of several analogous studies (e.g., Jacoby \& Dallas, 1981), was omitted in many others. These instructions may prompt subjects to handle implicit memory tasks like conventional recall or recognition tests. There is little evidence, however, supporting this type of argument. In fact, several investigators have noted that uninformed subjects realize early in the test session that some items come from the study list (e.g., Light \& Singh, 1987, p. 540; see also Oliphant, 1983, p. 395; Schacter, 1987, p. 510). Therefore, prior explicit information on this point should not introduce substantial changes, especially when there are several successive tasks having the same basic format. Such information may even be beneficial by reducing within- and between-subjects variability in strategy; preliminary observations indicated that uninformed subjects tended to be troubled when they discovered that some of the words to be found had previously been presented, and some subjects reported feeling unsure of having understood the initial instructions as the session proceeded.

According to one account of the action of explicit memory on implicit memory, subjects do not follow instructions to respond with the first word that comes to mind, but search deliberately for the target information in episodic memory. Although such a hypothesis is difficult to discount in absolute terms, it is worth noting that subjects consistently claim not to be using this kind of strategy. For example, Light and Singh (1987) reported that when questioned on this point after completing a word completion test, all of their subjects responded that they had not tried to use the word stems as retrieval cues. Informal observations on each of our four implicit memory tests led to similar conclusions.

The foregoing considerations concern only intentional or voluntary strategies. Schacter, in his recent critical review, argued for an alternative conceptualization of the action of explicit memory:

It is possible that some instances of what appear to be implicit memory may be better described as involuntary explicit memory: cases in which a test cue leads to an unintentional but fully conscious and explicit "reminding' of the occurrence of a prior episode.... At present, we know little about the relation between implicit memory and involuntary explicit memory, but future research and theorizing should be directed toward this issue. (Schacter, 1987, p. 510)

The results of the present work support the possibility that at least some apparent expressions of implicit memory may actually be expressions of involuntary explicit memory.

\section{Toward an Integrative Framework}

Associating the possibility of confusion between implicit and involuntary explicit memory with the hypotheses put forward above to account for dissociations between implicit memory measures may help to lay the groundwork for a more integrative approach. When the response is the product of an end-directed, step-by-step procedure, as is assumed to take place in perceptual clarification and word completion, all of the relevant information available to consciousness could be used; if some to-be-found words are presented during an early phase of the experiment, the explicit (although probably unintentional) recollection of these words may be used, for instance, to select a word from several plausible responses. When the response is accessed through some kind of direct, immediate processing, as in tachistoscopic identification and anagram solution, the remembering of initially presented words would be of no value, and the priming would be mediated by processes independent of explicit recall or recognition of words. Moreover, the trend toward a negative relationship between performance in perceptual 
clarification and performance in tachisotoscopic identification may be indicative of an active, analytic, problemsolving attitude that inhibits the action of genuine, unmediated priming, a hypothesis that is consistent with the results recently obtained by Peynircioglu and Watkins (1986).

The contention that explicit remembering of relevant information interferes with the completion of word fragments seems at first glance to be incompatible with studies on amnesic subjects that have cogently demonstrated that the priming effect in word completion is independent of recall or recognition (see the introduction). Tulving et al. (1982) also used word-fragment completion to show that the priming effect persisted intact when recognition was severely diminished by a long study-test interval. The discrepancy, however, is only apparent. For one thing, the factor structure of the word-completion test is less clearcut than the factor structure of perceptual clarification. Above all, we have only gone so far as to suggest that subjects may use explicit memory when dealing with a word-completion task; we do not claim that all subjects do so in all word-completion situations. It is quite possible, and even likely, that when the to-be-completed words are inaccessible explicitly, subjects deal with the task along different lines. Priming would then be a direct, unconsciously mediated phenomenon.

From a more general standpoint, the present results do not conflict with the hypothesis that implicit memory exists as a separate form of memory. Tasks such as tachistoscopic identification, and to a lesser extent anagram solution, may tap this form of memory in a relatively direct fashion. Other tasks may also provide reliable relevant information, so long as subjects have no direct access to the target information. However, when the latter condition is not fulfilled, data from tasks such as perceptual clarification and word completion may be strongly contaminated by explicit, although probably unintentional, remembering. To confirm and extend this a posteriori explanation, further investigation is required. In addition, it would be of great interest to study the relationships between other implicit memory tasks, such as lexical decision, and explicit memory.

\section{REFERENCES}

Carroll, M., Byrne, B., Kirsner, K. (1985). Autobiographical memory and perceptual learning: A developmental study using picture recognition, naming latency, and perceptual identification. Memory \& Cognition, 13, 273-279.

Carroll, M., Kirsner, K. (1982). Context and repetition effects in lexical decision and recognition memory. Joumal of Verbal Learning \& Verbal Behavior, 21, 55-69.

Clifton, C. (1966). Some determinants of the effectiveness of priming word associates. Journal of Verbal Learning \& Verbal Behavior. $5,167-171$.

Cofer, C. N. (1967). Conditions for the use of verbal associations. Psychological Bulletin, 68, 1-12.

Dannenbring, G. L., \& Briand, K. (1982). Semantic priming and the word repetition effect in a lexical decision task. Canadian Jour nal of Psychology, 36, 435-444.
Dark, V. J., Johnston, W. A., Mylfes-Worsley, M. . Farah, M. J. (1985). Levels of selection and capacity limits. Journal of Experimental Psychology: General, 114, 472-497.

Dominowski, R. L., Ekstrand, B. R. (1967). Direct and associative priming in anagram solving. Joumal of Experimental Psychology, 74. 84-86.

EicH, E. (1984). Memory for unattended events: Remembering with and without awareness. Memory \& Cognition, 12, 105-111.

Ellis, A. W., Young, A. W., Fiude, B. M., Hay, D. C. (1987). Repetition priming of face recognition. Quarterly Joumal of Experimental Psychology, 39A, 193-210

Feustel, T. C.. Shiffrin, R. M., Salasoo, A. (1983). Episodic and lexical contributions to the repetition effect in word identification. Journal of Experimental Psychology: General, 112, 337-339.

Forster, K. I. \& DAvIS, C. (1984). Repetition priming and frequency attenuation in lexical access. Journal of Experimental Psychology: Learning, Memory, \& Cognition, 10, 680-698.

Geiselman, R. E., Woodward, J. A., Beatty, J. (1982). Individual differences in verbal memory performance: $A$ test of alternative information-processing models. Joumal of Experimental Psychology: General, 111, 109-134.

Graf, P., Mandler, G. (1984). Activation makes words more accessible, but not necessarily more retrievable. Joumal of Verbal Leaming \& Verbal Behavior, 23, 553-568.

Graf, P., Mandler, G., \& Haden, M. (1982). Simulating amnesic symptoms in normal subjects. Science, 218, 1243-1244.

GraF, P.. SChacter, D. L. (1985). Implicit and explicit memory for new associations in normal and amnesic subjects. Joumal of Experimental Psychology: Leaming, Memory, \& Cognition, 11, 501-518.

Graf, P., Squire, L. R., Mandler, G. (1984). The information that amnesic patients do not forget. Joumal of Experimental Psychology: Learning, Memory, \& Cognition, 10, 164-178.

JaCOBY, L. L. (1983). Perceptual enhancement: Persistent effects of an experience. Journal of Experimental Psychology: Learning. Memory, \& Cognition, 9, 21-38.

JACOBY, L. L., \& DALLAS, M. (1981). On the relationship between autobiographical memory and perceptual learning. Joumal of Experimental Psychology: General, 110, 306-340.

JACOBY, L. L. \& WITHERSPOON, D. (1982). Remembering without awareness. Canadian Journal of Psychology, 36, 300-324.

Johnston, W. A., DARK, V. J., \& JACOBY, L. (1985). Perceprual fluency and recognition judgments. Journal of Experimental Psychology: Learning, Memory, \& Cognition, 11, 3-11.

KolERs, P. A (1976). Reading a year later. Journal of Experimental Psychology: Human Learning \& Memory, 2, 554-565.

Komatsu, S. (1985). Priming effects in the perceptual identification task after a long-term retention interval: In comparison with recognition memory. Japanese Journal of Psychology, 55, 362-365.

Light, L. L., Singh, A. (1987). Implicit and explicit memory in young and older adults. Journal of Experimental Psychology: Leaming, Memory, \& Cognition, 13,531-541.

Light, L. L., Singh, A., \& CAPPS, J. L. (1986). The dissociation of memory and awareness in young and older adults. Journal of Clinical \& Experimental Neuropsychology, 8, 62-74.

LOGAN, G. D. (1985). On the ability to inhibit simple thoughts and actions: II. Stop-signal studies of repetition priming. Joumal of Experimental Psychology: Learning, Memory, \& Cognition, 11, 675-691

LoRCH, R. F. (1986). Use of a word reading task for studying word recognition. Bulletin of the Psychonomic Sociery, 24, 11-14.

Moscovitch, M., Winocur, G., Mclachlan, D. (1986). Memory as assessed by recognition and reading time in normal and memoryimpaired people with Alzheimer's disease and other neurological disorders. Joumal of Experimental Psychology: General, , 115, 331-347.

NELSON, T, O. (1978). Detecting small amounts of information in memory: Savings for nonrecognized items. Journal of Experimental Psychology: Human Learning \& Memory, 4, 453-468.

Онта, N. (1984). The source of the long-term retention of priming effects. Behavioral \& Brain Sciences, 7, 249-250.

OLIPHANT, G. W. (1983). Repetition and recency effects in word recognition. Australian Journal of Psychology, 35, 393-403. 
Peynircioglu, Z. F., W W Wtkins, M. J. (1986). Cue depreciation: When word fragment completion is undermined by prior exposure to lesser fragments. Joumal of Experimental Psychology: Learning, Memory, \& Cognition, 12, 426-431.

Salasoo, A., Shiffrin, R. M., \& Feustel, T. C. (1985). Building permanent memory codes: Codification and repetition effects in word identification. Journal of Experimental Psychology: General, 114, $50-77$.

Scarborough, D. L., Cortese, C., \& Scarborough, H. S. (1977) Frequency and repetition effects in lexical memory. Journal of $E x-$ perimental Psychology: Human Perception \& Performance, 3, 1-12.

SCHACTER, D. L. (1987). Implicit memory: History and current status. Journal of Experimental Psychology: Leaming, Memory, \& Cognition, 13, 501-518.

Shimamura, A. P. (1985). Problems with the finding of stochastic independence as evidence for multiple memory systems. Bulletin of the Psychonomic Society, 23, 506-508.

SlamecKa, N. J. (1985). Ebbinghaus: Some rejoinders. Journal of Experimental Psychology: Leaming, Memory, \& Cognition, 11, 496-500.

Sloman, S. A., Hayman, C. A. G., Ohta, N., Law, J., \& Tulving, E. (1988). Forgetting in primed fragment completion. Journal of Experimental Psychology: Leaming, Memory, \& Cognition, 14, 223-239.
SQuiRe, L. R., * CohEN, N.J. (1984). Human memory and amnesia. In G. Lynch, J. McGaugh, \& N. M. Weinberger (Eds.), Neurobiology of learning and memory (pp. 3-64). New York: Guilford Press. TulviNG, E. (1976). Ecphoric processes in recall and recognition. In

J. Brown (Ed.), Recall and recognition (pp. 37-73). New York: Wiley. Tulving, E. (1985). How many memory systems are there? American Psychologist, 40, 385-398.

Tulving, E., Schacter, D., \& Stark, H. A. (1982). Priming effects in word-fragment completion are independent of recognition memory. Journal of Experimental Psychology: Learning, Memory, \& Cognition, 8, 352-373.

Underwood, B. J., Boruch, R. F., \&almi, R. A. (1978). Composition of episodic memory. Journal of Experimental Psychology: General, 107, 393-419.

Witkin, H. A., Oltman, P. K., Raskin, E., KarP, S. A. (1971). Manual for the Embedded Figures Tests. Palo Alto, CA: Consulting Psychologists Press.

(Manuscript received July 17,1987 revision accepted for publication March 12, 1988.) 